PROCEEDINGS OF THE

AMERICAN MATHEMATICAL SOCIETY

Volume 140, Number 6, June 2012, Pages 2141-2154

S 0002-9939(2011)11133-X

Article electronically published on October 24, 2011

\title{
POSITIVE CONSTRAINED MINIMIZERS FOR SUPERCRITICAL PROBLEMS IN THE BALL
}

\author{
MASSIMO GROSSI AND BENEDETTA NORIS
}

(Communicated by Matthew J. Gursky)

\begin{abstract}
We provide a sufficient condition for the existence of a positive solution to$$
-\Delta u+V(|x|) u=u^{p} \quad \text { in } B_{1},
$$

when $p$ is large enough. Here $B_{1}$ is the unit ball of $\mathbb{R}^{n}, n \geq 2$, and we deal with both Neumann and Dirichlet homogeneous boundary conditions. The solution turns out to be a constrained minimum of the associated energy functional. As an application we show that in case $V(|x|) \geq 0, V \not \equiv 0$ is smooth and $p$ is sufficiently large, and the Neumann problem always admits a solution.
\end{abstract}

\section{INTRODUCTION}

In this paper we study the existence of radial solutions to the following equation:

$$
\begin{cases}-\Delta u+V(|x|) u=u^{p} & \text { in } B_{1}, \\ u>0 & \text { in } B_{1},\end{cases}
$$

both with Neumann and Dirichlet homogeneous boundary conditions. Here $B_{1}$ is the unit ball of $\mathbb{R}^{n}, n \geq 2$, and $V(|x|) \geq 0, V \not \equiv 0$, is a smooth, radial function. We are interested when the exponent $p$ is large. Recent results (see [14, 8]) suggest that the existence of solutions of (1.1) is related to the critical points of a function $F(r)$, associated to this equation in the limit as $p \rightarrow+\infty$ (see (1.3) below). Our aim is to extend the known existence results in this direction through a better understanding of $F(r)$.

In order to be more precise, let us start by considering Neumann boundary conditions. We denote by $G(r, s)$ the Green function of the operator

$$
\mathcal{L} u=-u^{\prime \prime}-\frac{n-1}{r} u^{\prime}+V(r) u, \quad u^{\prime}(0)=0,
$$

with the Neumann boundary condition $u^{\prime}(1)=0$. Note that, unlike the case of higher dimensions, the Green function is bounded; hence $G(r, r)$ makes sense and we can define

$$
F(r)=\frac{\left|\partial B_{1}\right| r^{n-1}}{G(r, r)}, \quad r \in(0,1] .
$$

Here $\left|\partial B_{1}\right|$ is the measure of the boundary of the unit sphere. Note that we also define $F$ for $r=1$, meaning that $G(r, 1)$ is the punctual limit of $G(r, s)$ as $s \rightarrow 1$ (see Section 5 . $F(r)$ represents the energy naturally associated to the normalized Green

Received by the editors July 11, 2010 and, in revised form, February 13, 2011.

2010 Mathematics Subject Classification. Primary 35J25. 
function $G(\cdot, r) / G(r, r)$ (see Lemma 2.1). Our first result concerns the existence of solutions for the Neumann problem.

Theorem 1.1. Let us consider the problem

$$
\begin{cases}-\Delta u+V(|x|) u=u^{p} & \text { in } B_{1}, \\ u>0 & \text { in } B_{1}, \\ \frac{\partial u}{\partial \nu}=0 & \text { on } \partial B_{1},\end{cases}
$$

where $V(|x|) \geq 0, V \not \equiv 0$, is a smooth, radial function in $B_{1}$ such that $F(r)$ admits a local minimum point at $\bar{r} \in(0,1]$. Then, for $p$ large enough, there exists a radial solution $u_{p}=u_{p}(r)$ to (1.4) which verifies

$$
u_{p}(r) \rightarrow \frac{G(r, \bar{r})}{G(\bar{r}, \bar{r})} \quad \text { as } p \rightarrow+\infty \quad \text { in } H^{1}\left(B_{1}\right) \cap L^{\infty}\left(B_{1}\right) .
$$

We point out that we find as many different solutions as the number of local minimum points of $F(r)$ in $(0,1]$.

Moreover, $\bar{r}=1$ always being a local minimum point of $F(r)$ (see Section 5 ) we deduce the following result.

Theorem 1.2. Let $V(|x|) \geq 0, V \not \equiv 0$ be a smooth, radial function in $B_{1}$. Then, for $p$ large enough, there exists a radial solution $u_{p}=u_{p}(r)$ to (1.4) which converges to $G(r, 1) / G(1,1)$.

In particular, from (1.5), we derive the following new existence result for the problem with constant potential.

Corollary 1.3. Let $V(|x|) \equiv \lambda>0$. Then, for $p$ large enough, there exists a nonconstant radial solution $u_{p}=u_{p}(r)$ to (1.4).

These results continue the study of the supercritical case started in [13] and [14, with zero Dirichlet boundary conditions and $p$ large. However, here we have some important news. The first one concerns the technique used in the proof of Theorem 1.1] In [14, a crucial point in the construction of the solution was given by the following "limit problem":

$$
-U^{\prime \prime}=e^{U} \quad \text { in } \mathbb{R}
$$

and by the corresponding linearized equation. The solution was then found "close" to a projection of a suitable solution to (1.6). This approach is quite standard in this type of problem (there is a very wide literature on the topic) but involves heavy calculations. In the proof of Theorem 1.1 we do not use the limit problem (1.6), but we find the solution using some suitable constrained variational approach. Note that a similar idea was used in 21] to handle the supercritical problem. This technique, in the opinion of the authors, makes the proofs much simpler. Moreover, we think that similar ideas could be used in analogous problems with lack of compactness. Another important advantage resulting from this technique is that it does not require any nondegeneracy assumption on the minimum point $\bar{r}$ hypothesis, which is not easy to verify.

As it concerns Dirichlet boundary conditions, we partially recover, through this different technique, the results in [14. In fact the analogue of Theorem 1.1] holds in the following form. 
Theorem 1.4. Let us consider the problem

$$
\begin{cases}-\Delta u+V(|x|) u=u^{p} & \text { in } B_{1}, \\ u>0 & \text { in } B_{1}, \\ u=0 & \text { on } \partial B_{1},\end{cases}
$$

where $V(|x|) \geq 0, V \not \equiv 0$ is a smooth, radial function in $B_{1}$ such that $F(r)$ admits a local minimum point at $\bar{r} \in(0,1)$. Then, for $p$ large enough, there exists a radial solution $u_{p}=u_{p}(r)$ to (1.7) which verifies

$$
u_{p}(r) \rightarrow \frac{G(r, \bar{r})}{G(\bar{r}, \bar{r})} \quad \text { as } p \rightarrow+\infty \quad \text { in } H^{1}\left(B_{1}\right) \cap L^{\infty}\left(B_{1}\right) .
$$

Of course, in this case, the term $G(r, s)$ appearing in (1.3) and (1.8) is the Green function of the operator (1.2) with the Dirichlet boundary condition $u(1)=0$. The proof of Theorem 1.4 is the same as the one of Theorem 1.1 (it is even easier because we do not need to analyze the case $\bar{r}=1$ ). For this reason we omit it. Actually, our technique gives a unified proof for both Dirichlet and Neumann boundary conditions. Moreover, again in the Dirichlet case, Catrina proved in 8 that the condition in Theorem 1.4 is "almost" necessary. Indeed he proved the following result.

Theorem 1.5. If the function $F_{p}(r)=\frac{\frac{p-1}{p+3}(n-1)}{G(r, r)}$ is monotonic and nonconstant, then problem (1.7) has no solution.

Since $F_{p} \rightarrow F$ uniformly in any interval $\left[r_{0}, 1\right]$ as $p \rightarrow+\infty$, we have that the existence of a minimum to the function $F$ becomes "almost" necessary for the existence of a solution.

We end this section with a brief history of the problem (1.1). First, if $1<p<$ $\frac{n+2}{n-2}$ for $n \geq 3$ (the subcritical case) and $p>1$ if $n=2$, it is not difficult to prove the existence of a solution. This can be shown observing that the infimum

$$
S_{p}=\inf \left\{\int_{\Omega}\left(|\nabla u|^{2}+V(x) u^{2}\right): \int_{\Omega}|u|^{p+1}=1, u \in H^{1}(\Omega)\right\}
$$

is achieved because of the compact embedding of $H^{1}(\Omega)$ in $L^{p+1}(\Omega)$ (the same holds for $H_{0}^{1}(\Omega)$ ).

If $p=\frac{n+2}{n-2}$ for $n \geq 3$ (the critical case), it is well known that the existence of a solution to (1.1) depends on the shape of $\Omega$ and on the properties of $V(x)$. Since there is a huge literature on this topic we just mention the pioneering papers by Brezis and Nirenberg [7] and Bahri and Coron [6] for the Dirichlet case. The Neumann problem (1.1) was first studied when $V(x)$ is a positive constant $\lambda$, and some existence results were established in $19,9,4,5,20,2$. See also 1,3 for the case where $V$ is not constant.

The supercritical case $p>\frac{n+2}{n-2}$ is much more difficult to handle since there is no embedding of $H^{1}(\Omega)$ in $L^{p+1}(\Omega)$. A consequence of this fact is that the infimum $S_{p}$ in (1.9) is zero, and hence it cannot be used to find a solution to (1.1). Some interesting existence and nonexistence results in special domains with Dirichlet boundary conditions are due to Passaseo $(21,22])$. We also mention the recent paper [10] concerning domains with small circular holes. We emphasize that the case of a general domain seems not yet fully understood. Regarding the case of the ball, to our knowledge the only results are those of [14] (already mentioned above) and 17, 18, (here $V$ is constant). 
Finally, let us mention some interesting results in the whole space contained in 15.

Unlike the Dirichlet case, where in recent years there have been several developments, in the Neumann case there is a very poor literature. To our knowledge the only results in the supercritical case are due to $\mathrm{Ni}$ (see [19]) and Lin-Ni (see [16]). In particular the authors prove the following.

Theorem 1.6. Let us consider the problem

$$
\begin{cases}-\Delta u+\lambda u=u^{p} & \text { in } B_{1}, \\ u>0 & \text { in } B_{1}, \\ \frac{\partial u}{\partial \nu}=0 & \text { on } \partial B_{1} .\end{cases}
$$

Then, there exist positive constants $\lambda_{0}=\lambda_{0}(n, p)$ and $\lambda_{1}=\lambda_{1}(n)$ such that

i) for any $\lambda>\lambda_{1}$ there exists at least a nonconstant radial solution to (1.4),

ii) for any $\lambda<\lambda_{0}$ (1.4) does not admit any nonconstant radial solution.

From this result and Corollary 1.3, we derive that the constant $\lambda_{0}(n, p) \rightarrow 0$ as $p \rightarrow+\infty$.

The paper is organized as follows. Sections 2 4 contain the proof of Theorem 1.1 in the case $\bar{r} \in(0,1)$. In Section 2 we introduce a family of variational problems depending on a parameter $p \in(1, \infty)$ and a limit problem. The existence and convergence of the minimizers is shown in Section 3. In Section 4 we end the proof of Theorem 1.1 and present an additional property of the solution (see Proposition 4.3). Section 5 deals with the case $\bar{r}=1$ and with Theorem 1.2. Finally, in the Appendix we collect some properties of the Green function.

\section{VARIATIONAL SETTING AND NOTATION}

We introduce the Sobolev space of radial functions

$$
H_{r}^{1}\left(B_{1}\right)=\left\{u \in H^{1}\left(B_{1}\right): u=u(|x|)\right\} .
$$

In the following we will often abuse the notation $u(r)=u(|x|)$. We find solutions to (1.4) as constrained minimizers (in this space) of the energy functional

$$
Q(u)=\int_{B_{1}}\left[|\nabla u|^{2}+V(|x|) u^{2}\right],
$$

under the standard $L^{p}$-mass constraint and under an additional constraint which will be proven to be natural for $p$ sufficiently large. Let $\bar{r} \in(0,1)$ be a local minimum point of $F(r)$; then there exist $0<R_{1}<R_{2}<1$ such that $\bar{r}$ is a global minimum point in $\left[R_{1}, R_{2}\right]$. Set

$K_{p}=\left\{u \in H_{r}^{1}\left(B_{1}\right):\left(\left|B_{1}\right|^{-1} \int_{B_{1}}|u|^{p+1}\right)^{\frac{1}{p+1}}=1\right.$ and $|u| \leq c$ in $\left.B_{R_{1}} \cup\left(B_{1} \backslash B_{R_{2}}\right)\right\}$,

where $B_{R}$ denotes the ball centered at the origin of radius $R$ and $c$ satisfies

$$
\max \left\{\frac{G\left(R_{1}, \bar{r}\right)}{G(\bar{r}, \bar{r})}, \frac{G\left(R_{2}, \bar{r}\right)}{G(\bar{r}, \bar{r})}\right\}<c<1
$$

Notice that by the strong maximum principle, for every $r \neq s$ it holds that $G(r, s)<$ $G(s, s)$; hence it is always possible to find $c$ as in (2.3). We consider the following infimum:

$$
J_{p}=\inf \left\{Q(u), u \in K_{p}\right\} .
$$


Of course, a nonnegative function which achieves $J_{p}$ provides a solution (up to a multiplicative constant) to (1.4) if $u<c$ in $B_{R_{1}} \cup\left(B_{1} \backslash B_{R_{2}}\right)$. Actually, we will see that the condition $|u| \leq c$ in $B_{R_{1}}$ prevents the solutions from concentrating around the origin and $|u| \leq c$ in $B_{1} \backslash B_{R_{2}}$ forces the solution to have its maximum around the local minimum point $\bar{r}$.

Our strategy is based on the asymptotic analysis of the minimization problem $J_{p}$ as $p \rightarrow \infty$. In fact, we will show the convergence of $J_{p}$ to the following infimum

$$
J_{\infty}=\inf \left\{Q(u), u \in K_{\infty}\right\}
$$

where $K_{\infty}$ is

$$
K_{\infty}=\left\{u \in H_{r}^{1}\left(B_{1}\right):\|u\|_{\infty}=1 \text { and }|u| \leq c \text { in } B_{R_{1}} \cup\left(B_{1} \backslash B_{R_{2}}\right)\right\} .
$$

A key property is the following lemma, which enlightens our choice of the constant $c$ in (2.3).

Lemma 2.1. For every $r \in(0,1)$ it holds that

$$
F(r)=Q\left(\frac{G(\cdot, r)}{G(r, r)}\right) .
$$

Proof. By passing to polar coordinates we obtain

$$
Q\left(\frac{G(\cdot, r)}{G(r, r)}\right)=\frac{\left|\partial B_{1}\right|}{G(r, r)^{2}} \int_{0}^{1}\left[\left(\frac{\partial G(t, r)}{\partial t}\right)^{2}+V(t) G(t, r)^{2}\right] t^{n-1} d t .
$$

On the other hand, by the definition of the Green function, we have

$$
-\frac{\partial^{2} G(t, r)}{\partial t^{2}}-\frac{n-1}{t} \frac{\partial G(t, r)}{\partial t}+V(t) G(t, r)=\delta_{r}
$$

in the sense of distributions. Multiplying the last equation by $t^{n-1} G(t, r)$, integrating over $(0,1)$ and finally substituting in (2.8), we obtain the thesis.

\section{Existence And CONVERGEnCE OF THE CONSTRAined Minimizers}

Let us start by proving the existence of a minimizer to $J_{p}$.

Proposition 3.1. There exists a nonnegative function $u_{p} \in K_{p}$ such that $J_{p}=$ $Q\left(u_{p}\right)$.

Proof. Let $u_{p}^{n} \in K_{p}$ be a minimizing sequence for $J_{p}$; that is,

$$
\lim _{n \rightarrow \infty} Q\left(u_{p}^{n}\right)=J_{p} \text {. }
$$

Being $V(|x|) \geq 0, V \not \equiv 0$, we infer that $\left\{u_{p}^{n}\right\}_{n}$ is bounded in $H_{r}^{1}\left(B_{1}\right)$. Hence it converges weakly (up to subsequences) to some function $u_{p} \in H_{r}^{1}\left(B_{1}\right)$ and almost everywhere in $B_{1}$. Now, it is immediate to check that $u_{p} \in K_{p}$, since

$$
\left\|u_{p}^{n}-u_{p}\right\|_{L^{p}\left(B_{1}\right)}=\left\|u_{p}^{n}-u_{p}\right\|_{L^{p}\left(B_{R_{1}}\right)}+\left\|u_{p}^{n}-u_{p}\right\|_{L^{p}\left(B_{1} \backslash B_{R_{1}}\right)}
$$

and both terms tend to zero (as $n \rightarrow \infty$ ): the first one, since $\left|u_{p}^{n}\right| \leq c$, by the Lebesgue convergence theorem and the second one by the compact embedding of $H_{r}^{1}\left(B_{1} \backslash B_{R_{1}}\right)$ into $L^{p}\left(B_{1} \backslash B_{R_{1}}\right)$ for every $p$. Finally, $u_{p}$ can be chosen nonnegative since $\int_{B_{1}}|\nabla| u||^{2}=\int_{B_{1}}|\nabla u|^{2}$.

We are in a position to prove the weak convergence of the minimizers as $p \rightarrow \infty$. Let $A=B_{R_{2}} \backslash B_{R_{1}}$. 
Lemma 3.2. Let $u_{p_{n}} \in K_{p_{n}}$ be a sequence of minimizers to $J_{p_{n}}$. Then there exists $u_{\infty} \in H_{r}^{1}\left(B_{1}\right)$ such that, up to a subsequence denoted again by $p_{n}$, it holds that

$$
u_{p_{n}} \rightarrow u_{\infty} \text { in } H^{1}\left(B_{1}\right), \quad u_{p_{n}} \rightarrow u_{\infty} \quad \text { in } L^{q}\left(B_{1}\right), \forall q<\infty .
$$

In addition, $u_{p_{n}} \rightarrow u_{\infty}$ in $L^{\infty}(A)$.

Proof. Let us prove that the sequence $J_{p_{n}}$ is bounded in $H^{1}\left(B_{1}\right)$. Then the statement follows, proceeding as in the proof of Proposition 3.1. To this aim consider a nonnegative test function $\eta \in K_{1}$ and set

$$
\eta_{p}=\frac{\eta}{\left(\left|B_{1}\right|^{-1} \int_{B_{1}} \eta^{p+1}\right)^{\frac{1}{p+1}}} .
$$

Using the Hölder inequality we obtain, for every $p \geq 1$,

$$
Q\left(\eta_{p}\right)=\frac{Q(\eta)}{\left(\left|B_{1}\right|^{-1} \int_{B_{1}} \eta^{p+1}\right)^{\frac{2}{p+1}}} \leq \frac{Q(\eta)}{\left|B_{1}\right|^{-1} \int_{B_{1}} \eta^{2}}=Q(\eta) .
$$

Being $\eta_{p} \in K_{p}$, this implies $J_{p} \leq Q(\eta)$, which concludes the proof.

The next lemma, roughly speaking, ensures that the "mass" of the $u_{p}$ 's concentrates in $A$ as $p \rightarrow \infty$.

Lemma 3.3. It holds that

$$
\gamma_{p}=\left(|A|^{-1} \int_{A} u_{p}^{p+1}\right)^{\frac{1}{p+1}} \rightarrow 1 \quad \text { as } p \rightarrow \infty .
$$

Proof. Since $u_{p} \in K_{p}$, a direct calculation gives

$$
\gamma_{p}=|A|^{-\frac{1}{p+1}}\left(\left|B_{1}\right|-\int_{B_{1} \backslash A} u_{p}^{p+1}\right)^{\frac{1}{p+1}} .
$$

Now, $\int_{B_{1} \backslash A} u_{p}^{p+1} \leq \int_{B_{1} \backslash A} c^{p+1} \rightarrow 0$ (being $c<1$ ); hence $\gamma_{p} \rightarrow 1$.

As a consequence of the previous lemma we deduce that $u_{\infty} \in K_{\infty}$. In fact, the following holds.

Lemma 3.4. The limit function $u_{\infty}$ satisfies $\left\|u_{\infty}\right\|_{L^{\infty}(A)}=1$.

Proof. Fix $p>1$. Then, the Hölder inequality gives $\left(\gamma_{p}\right.$ is defined in Lemma 3.3)

$$
\lim _{q \rightarrow \infty}\left(|A|^{-1} \int_{A} u_{p}^{q+1}\right)^{\frac{1}{q+1}} \geq\left(|A|^{-1} \int_{A} u_{p}^{p+1}\right)^{\frac{1}{p+1}}=\gamma_{p} .
$$

By Lemma 3.2 we have that $u_{p} \rightarrow u_{\infty}$ in $L^{\infty}(A)$, and then

$$
\left\|u_{\infty}\right\|_{L^{\infty}(A)}=\lim _{p \rightarrow \infty}\left\|u_{p}\right\|_{L^{\infty}(A)}=\lim _{p \rightarrow \infty} \lim _{q \rightarrow \infty}\left(|A|^{-1} \int_{A} u_{p}^{q+1}\right)^{\frac{1}{q+1}} \geq \lim _{p \rightarrow \infty} \gamma_{p}=1 .
$$

Similarly we compute, for a fixed $q>1$,

$$
\lim _{p \rightarrow \infty}\left(|A|^{-1} \int_{A} u_{p}^{q+1}\right)^{\frac{1}{q+1}} \leq \lim _{p \rightarrow \infty}\left(|A|^{-1} \int_{A} u_{p}^{p+1}\right)^{\frac{1}{p+1}}=1,
$$


which gives the opposite inequality,

$$
\left\|u_{\infty}\right\|_{L^{\infty}(A)}=\lim _{q \rightarrow \infty}\left\|u_{\infty}\right\|_{L^{q}(A)}=\lim _{q \rightarrow \infty} \lim _{p \rightarrow \infty}\left(|A|^{-1} \int_{A} u_{p}^{q+1}\right)^{\frac{1}{q+1}} \leq 1 .
$$

On the other hand we have the following approximation result.

Lemma 3.5. Let $u \in K_{\infty}$ be a nonnegative function. Then, for any $p>1$, there exists $w_{p} \in K_{p}$ such that $w_{p} \rightarrow u$ in $H^{1}\left(B_{1}\right)$ as $p \rightarrow+\infty$.

Proof. Let $\varphi(x)=\min (u(x), c)$, defined in $A$. For $\sigma \geq 0$, we define

$$
w_{\sigma}= \begin{cases}u & \text { in } B_{1} \backslash A, \\ \sigma(u-\varphi)+u & \text { in } A .\end{cases}
$$

Let us show that for any $p>1$, there exists $\sigma_{p}>0$ such that $w_{\sigma} \in K_{p}$. To this aim we introduce the function

$$
Z(\sigma)=\int_{B_{1}}\left|w_{\sigma}\right|^{p+1}=\left|B_{1}\right|^{-1} \int_{B_{1} \backslash A}|u|^{p+1}+\left|B_{1}\right|^{-1} \int_{A}|\sigma(u-\varphi)+u|^{p+1} .
$$

$Z$ is continuous, $\lim _{\sigma \rightarrow \infty} Z(\sigma)=+\infty$, and moreover

$$
Z(0)=\left|B_{1}\right|^{-1} \int_{B_{1}}|u|^{p+1}<\left\|u_{p}\right\|_{L^{\infty}\left(B_{1}\right)}=1,
$$

since $u \not \equiv 1$, by the definition of $K_{\infty}$. Hence there exists $\sigma_{p}>0$ such that $Z\left(\sigma_{p}\right)=1$. Setting $w_{p}=w_{\sigma_{p}}$ and observing that $w_{p}$ is continuous in $B_{1}$ and $w_{p} \in H^{1}\left(B_{1}\right)$, we conclude that $w_{p} \in K_{p}$. Moreover, since $w_{p} \leq c$ in $B_{1} \backslash A$, we have

$$
\left(|A|^{-1} \int_{A}\left|w_{p}\right|^{p+1}\right)^{\frac{1}{p+1}} \rightarrow 1 \quad \text { as } p \rightarrow \infty .
$$

Let us prove that $\sigma_{p} \rightarrow 0$, which concludes the proof. If not, there exists $\delta>0$ such that $\sigma_{p}>\delta$ for every $p$. This implies $\sigma_{p}(u-\varphi) \geq \delta(u-\varphi)$ and hence $\left(w_{p}\right.$ being nonnegative)

$$
\begin{aligned}
1 & =\lim _{p \rightarrow \infty}\left(|A|^{-1} \int_{A}\left|w_{p}\right|^{p+1}\right)^{\frac{1}{p+1}} \geq \lim _{p \rightarrow \infty}\left(|A|^{-1} \int_{A}|(1+\delta) u-\delta \varphi|^{p+1}\right)^{\frac{1}{p+1}} \\
& =\|(1+\delta) u-\delta \varphi\|_{L^{\infty}(A) .}
\end{aligned}
$$

Now let $\hat{r} \in\left(R_{1}, R_{2}\right)$ be such that $u(\hat{r})=1$. Then we have

$1 \geq\|(1+\delta) u-\delta \varphi\|_{L^{\infty}(A)} \geq(1+\delta) u(\hat{r})-\delta \varphi(\hat{r}) \geq(1+\delta)-\delta c=1+\delta(1-c)>1$,

which is a contradiction.

The next proposition proves the convergence of the constrained variational problems $J_{p}$ to the limit problem $J_{\infty}$ (see (2.5)).

Proposition 3.6. We have that

$$
\lim _{p \rightarrow \infty} J_{p}=J_{\infty} \quad \text { and } \quad u_{p} \rightarrow u_{\infty} \text { in } H^{1}\left(B_{1}\right) .
$$

Moreover, $u_{\infty} \in K_{\infty}$ and $Q\left(u_{\infty}\right)=J_{\infty}$. 
Proof. We already know that $u_{p} \rightarrow u_{\infty}$ in $H^{1}\left(B_{1}\right)$ (Lemma 3.2) and that $u_{\infty} \in K_{\infty}$ (Lemma 3.4). On one hand we have

$$
J_{\infty} \leq Q\left(u_{\infty}\right) \leq \liminf _{p \rightarrow \infty} Q\left(u_{p}\right)=\liminf _{p \rightarrow \infty} J_{p}
$$

where we used the lower semicontinuity of the $H^{1}$-norm with respect to weak convergence. In order to prove the reverse inequality, let $u \in K_{\infty}, u \geq 0$, and let $w_{p}$ be the corresponding approximating sequence founded in Lemma 3.5. Then it holds that

$$
Q(u)=\lim _{p \rightarrow \infty} Q\left(w_{p}\right) \geq \limsup _{p \rightarrow \infty} J_{p} .
$$

Since $J_{\infty}$ can be equivalently characterized as $\inf \left\{Q(u): u \in K_{\infty}, u \geq 0\right\}$, we have obtained that $J_{p} \rightarrow J_{\infty}$. As a consequence, the inequalities in (3.15) are in fact equalities, which implies $Q\left(u_{\infty}\right)=J_{\infty}$ and also the $H^{1}$-strong convergence.

\section{Proof of Theorem 1.1 when $\bar{r} \in(0,1)$}

The variational characterization of $u_{\infty}$ proved in the previous section allows us to derive the following.

Lemma 4.1. There exists a unique $r_{\infty} \in\left[R_{1}, R_{2}\right]$ such that $u_{\infty}\left(r_{\infty}\right)=1$. Moreover, $u_{\infty}(r)$ solves

$$
-u^{\prime \prime}-\frac{n-1}{r} u^{\prime}+V(r) u=0, \quad u^{\prime}(0)=0
$$

in $\left(0, R_{1}\right) \cup\left(R_{1}, r_{\infty}\right) \cup\left(r_{\infty}, R_{2}\right) \cup\left(R_{2}, 1\right)$.

Proof. Let us first show that $u_{\infty}$ solves the equation in $\left(0, R_{1}\right)$. To this aim let $w$ be the solution of

$$
\left\{\begin{array}{l}
-w^{\prime \prime}-\frac{n-1}{r} w^{\prime}+V(r) w=0 \\
w^{\prime}(0)=0, w\left(R_{1}\right)=u_{\infty}\left(R_{1}\right) .
\end{array} \quad \text { in }\left(0, R_{1}\right),\right.
$$

Hence $w$ minimizes the functional $Q(u)$ in $B_{R_{1}}$. Moreover the maximum principle ensures

If we define

$$
0<w<c \text { in } B_{R_{1}} \text {. }
$$

$$
\widetilde{w}= \begin{cases}w & \text { in } B_{R_{1}}, \\ u_{\infty} & \text { in } B_{1} \backslash B_{R_{1}},\end{cases}
$$

then $\widetilde{w} \in K_{\infty}$ and $Q(\widetilde{w}) \leq Q\left(u_{\infty}\right)$. This implies that $\widetilde{w} \equiv u_{\infty}$ in $B_{R}$, and so $u_{\infty}$ solves (4.1) in $B_{R_{1}}$. One can proceed similarly in $B_{1} \backslash B_{R_{2}}$.

Set $X=\left\{r \in\left[R_{1}, R_{2}\right]: u_{\infty}(r)=1\right\}$ and let $r_{m}=\inf X, r_{M}=\sup X(X$ is not empty since $\left.\left\|u_{\infty}\right\|_{L^{\infty}(A)}=1\right)$. Notice that $u_{\infty}$ solves (4.1) in the open set $\left[R_{1}, R_{2}\right] \backslash X$, since here the function does not touch the obstacle. In particular, by the maximum principle, the interval $\left[r_{m}, r_{M}\right]$ is contained in $X$. Let us end the proof by showing that $X$ is a singleton. By contradiction assume that this is not true; let $w$ be the solution of

$$
\left\{\begin{array}{l}
-w^{\prime \prime}-\frac{n-1}{r} w^{\prime}+V(r) w=0 \\
u\left(R_{1}\right)=u_{\infty}\left(R_{1}\right), u\left(r_{M}\right)=1 .
\end{array} \quad \text { in }\left(R_{1}, r_{M}\right),\right.
$$

Since $u_{\infty} \equiv 1$ in $\left(r_{m}, r_{M}\right)$, whereas $w$ cannot be constant in an interval by the strong maximum principle, we have that $Q(w)<Q\left(u_{\infty}\right)$ in the annulus $B_{r_{M}} \backslash B_{R_{1}}$, which again leads to a contradiction as before. Thus $X$ is a singleton. 
We deduce that $u_{\infty}(r)$ is regular in $A$, except for the point $r=r_{\infty}$, with different right and left derivatives. In the next proposition we show that $r_{\infty}=\bar{r}$ (recall that $\bar{r}$ is a local minimum point of $F$ ) and that $u_{\infty}$ coincides in fact with the normalized Green function $G(r, \bar{r})$.

Proposition 4.2. We have that

$$
u_{\infty}(r)=\frac{G(r, \bar{r})}{G(\bar{r}, \bar{r})} .
$$

Proof. Let us first prove that $Q\left(u_{\infty}\right) \geq F\left(r_{\infty}\right)$, with $r_{\infty}$ given by the previous lemma. To this aim we consider the auxiliary problem

$$
\inf \left\{Q(u): u \in H_{r}^{1}\left(B_{1}\right), 0 \leq u \leq 1 \text { in } B_{1}, u\left(r_{\infty}\right)=1\right\} .
$$

Arguing as in the previous lemma, we have that the function which achieves (4.6) solves the problem

$$
\begin{cases}-u^{\prime \prime}-\frac{n-1}{r} u^{\prime}+V(r) u=0 & \text { in }\left(0, r_{\infty}\right) \cup\left(r_{\infty}, 1\right), \\ u^{\prime}(0)=0, u\left(r_{\infty}\right)=1, u^{\prime}(1)=0, u \in H_{r}^{1}\left(B_{1}\right) . & \end{cases}
$$

Since the function $G\left(r, r_{\infty}\right) / G\left(r_{\infty}, r_{\infty}\right)$ satisfies (4.7), then we derive that it minimizes (4.6). Moreover, $u_{\infty}$ belongs to the minimization set in (4.6); hence

$$
Q\left(u_{\infty}\right) \geq Q\left(\frac{G\left(\cdot, r_{\infty}\right)}{G\left(r_{\infty}, r_{\infty}\right)}\right)=F\left(r_{\infty}\right)
$$

(the last equality comes from Lemma 2.1). Note that at this stage we still do dot know whether $G\left(r, r_{\infty}\right) / G\left(r_{\infty}, r_{\infty}\right) \in K_{\infty}$.

Next we show that $r_{\infty}=\bar{r}$. Assume not. We know that $r_{\infty} \in\left[R_{1}, R_{2}\right]$ and that $F(\bar{r})<F(r) \forall r \in\left[R_{1}, R_{2}\right]$, since $\bar{r}$ is a strict local minimum point (see Lemma 6.3 in the Appendix). Hence

$$
Q\left(u_{\infty}\right) \geq F\left(r_{\infty}\right)>F(\bar{r})=Q\left(\frac{G(\cdot, \bar{r})}{G(\bar{r}, \bar{r})}\right) .
$$

Now, due to our choice of the constant $c$, we have that $G(r, \bar{r}) / G(\bar{r}, \bar{r}) \in K_{\infty}$; hence the last inequality gives a contradiction. We conclude that $r_{\infty}=\bar{r}$ and, in turn, that $u_{\infty}=G(r, \bar{r}) / G(\bar{r}, \bar{r})$.

Proof Theorem 1.1 when $\bar{r} \in(0,1)$. Let us consider the function $u_{p}$ which minimizes $J_{p}$. Then, proceeding exactly as in Lemma 2.6 in [21, we have that

$$
-\Delta u_{p}+V(|x|) u_{p} \leq \lambda_{p} u_{p}^{p} \quad \text { in } B_{1}
$$

and

$$
-\Delta u_{p}+V(|x|) u_{p}=\lambda_{p} u_{p}^{p} \text { in } A,
$$

where $\lambda_{p}$ is a Lagrange multiplier. We want to show that $\left|\lambda_{p}\right| \leq C$, where $C$ is a positive constant independent of $p$. First note that multiplying (4.10) by $u_{p}$ and integrating in $B_{1}$ we immediately obtain that $\lambda_{p}$ is positive. In order to get a bound from above to $\lambda_{p}$, let us choose $R_{1}<\bar{R}_{1}<r_{\infty}<\bar{R}_{2}<R_{2}$ such that $\int_{\partial B_{\bar{R}_{1}}}\left|\nabla u_{p}\right|^{2}$ and $\int_{\partial B_{\bar{R}_{2}}}\left|\nabla u_{p}\right|^{2}$ are uniformly bounded in $p$ (this is possible since $\int_{B_{1}}\left|\nabla u_{p}\right|^{2}$ is 
uniformly bounded). Then multiply (4.11) by $u_{p}$ and integrate in $\bar{A}=B_{\bar{R}_{2}} \backslash B_{\bar{R}_{1}}$. We get

$$
\lambda_{p} \int_{\bar{A}}\left|u_{p}\right|^{p+1} \leq J_{p}+\int_{\partial \bar{A}} \frac{\partial u_{p}}{\partial \nu} u_{p} \leq J_{p}+\left(\int_{\partial \bar{A}}\left|\nabla u_{p}\right|^{2}\right)^{\frac{1}{2}}\left(\int_{\partial \bar{A}}\left|u_{p}\right|^{2}\right)^{\frac{1}{2}} .
$$

Now, proceeding as in Lemma 3.3. one can show that $\int_{\bar{A}} u_{p}^{p+1} \rightarrow 1$ as $p \rightarrow \infty$ (since $\bar{R}_{1}<r_{\infty}<\bar{R}_{2}$ ). Hence (4.12) implies that $\lambda_{p} \leq C$, for some constant $C$ independent of $p$.

Finally we claim that

$$
u_{p} \rightarrow u_{\infty} \quad \text { as } p \rightarrow \infty \text { in } L^{\infty}\left(B_{1}\right) .
$$

Notice first that $u_{p} \rightarrow u_{\infty}$ in $L^{\infty}\left(B_{1} \backslash B_{\epsilon}\right)$ for any $\epsilon>0$, because of the embedding of $H_{r}^{1}\left(B_{1} \backslash B_{\epsilon}\right)$ into $L^{\infty}\left(B_{1} \backslash B_{\epsilon}\right)$. Recalling the equation satisfied by $u_{\infty}$ in $B_{R_{1}}$, we get from (4.10) that

$$
-\Delta\left(u_{p}-u_{\infty}\right)+V(x)\left(u_{p}-u_{\infty}\right) \leq \lambda_{p} u_{p}^{p} \quad \text { in } B_{R_{1}} .
$$

By known regularity results (see for example Theorem 9.1 in [12]), we have that

$$
\left\|u_{p}-u_{\infty}\right\|_{L^{\infty}\left(B_{R_{1}}\right)} \leq\left\|u_{p}-u_{\infty}\right\|_{L^{\infty}\left(\partial B_{R_{1}}\right)}+C\left\|\lambda_{p} u_{p}^{p}\right\|_{L^{n}\left(B_{R_{1}}\right)} .
$$

Being $u_{p}<1$ in $B_{R_{1}}$ and $\left\{\lambda_{p}\right\}$ a bounded sequence, we deduce that the right hand side in the previous inequality converges to zero as $p \rightarrow \infty$; hence (4.13) is proved.

Now, as a consequence of Proposition 4.2 of the uniform convergence and of the choice of $c$ in (2.3), we deduce that

$$
u_{p}<c \text { in } B_{R_{1}} \cup\left(B_{1} \backslash B_{R_{2}}\right) .
$$

Hence $u_{p}$ solves

$$
-\Delta u_{p}+V(|x|) u_{p}=\lambda_{p} u_{p}^{p} \quad \text { in } B_{1},
$$

for a Lagrange multiplier $\lambda_{p}>0$. A suitable multiple of $u_{p}$ provides a solution to (1.4).

With $\bar{r}$ being a critical point of $F(r)$, we have that the function $G(r, \bar{r})$ verifies the following interesting reflection principle.

Proposition 4.3. We have that

$$
\lim _{r \rightarrow \bar{r}^{-}} G_{r}(r, \bar{r})=-\lim _{r \rightarrow \bar{r}^{+}} G_{r}(r, \bar{r})=\frac{1}{2},
$$

where $G_{r}(r, s)$ denotes the derivative with respect to $r$.

Proof. Lemma 6.2 gives $F(r)=\left|\partial B_{1}\right| /[\xi(r) \zeta(r)]$. Hence, $\bar{r}$ being a critical point of $F(r)$, we have

$$
\xi^{\prime}(\bar{r}) \zeta(\bar{r})+\xi(\bar{r}) \zeta^{\prime}(\bar{r})=0
$$

On the other hand, again by Lemma 6.2 we have that

$$
\lim _{r \rightarrow \bar{r}^{-}} G_{r}(r, \bar{r})=\bar{r}^{n-1} \xi^{\prime}(\bar{r}) \zeta(\bar{r}), \quad \lim _{r \rightarrow \bar{r}^{+}} G_{r}(r, \bar{r})=\bar{r}^{n-1} \xi(\bar{r}) \zeta^{\prime}(\bar{r}),
$$

which, together with (4.19), gives the first equality. In order to obtain the value of the left derivative it is enough to combine (4.19) with (6.2) at $\bar{r}$. 


\section{THE CASE $\bar{r}=1$}

In this section we conclude the proof of Theorem 1.1 dealing with the case $\bar{r}=1$, and we prove Theorem 1.2. Let us start by showing that Lemma 2.1 still holds at $r=1$. Recall that $G(r, 1)$ is well defined (Lemma 6.2 and Lemma 6.1) and that it is the punctual limit of $G(r, s)$ as $s \rightarrow 1$.

Lemma 5.1. We have that

$$
F(1)=Q\left(\frac{G(\cdot, 1)}{G(1,1)}\right)
$$

Proof. It comes from Lemma 6.2 that $G(r, 1) / G(1,1)$ is well defined (since $\zeta(1) \neq$ 0 ), and moreover $G(r, 1) / G(1,1)=\xi(r) / \xi(1)$. In order to evaluate the energy of this function, let us write the equation satisfied by $\xi$ and multiply by $r^{n-1} \xi$. We have

$$
V(r) \xi^{2} r^{n-1}=r^{n-1} \xi^{\prime \prime} \xi+(n-1) r^{n-2} \xi^{\prime} \xi=\frac{d}{d r}\left(r^{n-1} \xi^{\prime} \xi\right)-r^{n-1}\left(\xi^{\prime}\right)^{2},
$$

and hence

$$
\int_{0}^{1}\left[\left(\xi^{\prime}\right)^{2}+V(r) \xi^{2}\right] r^{n-1} d r=\xi^{\prime}(1) \xi(1) .
$$

As a consequence it holds that

$$
Q\left(\frac{G(\cdot, 1)}{G(1,1)}\right)=\frac{\left|\partial B_{1}\right| \xi^{\prime}(1)}{\xi(1)}=\frac{\left|\partial B_{1}\right| \xi^{\prime}(1) \zeta(1)}{\xi(1) \zeta(1)}=\frac{\left|\partial B_{1}\right|}{\xi(1) \zeta(1)}=F(1),
$$

where we used (6.2) at $r=1$ and that $\zeta^{\prime}(1)=0$.

Proof of Theorem 1.1 when $\bar{r}=1$. This is analogous to the case $\bar{r} \in(0,1)$. Choose $R_{1}$ and $c$ in such a way that 1 is a global minimum point in $\left[R_{1}, 1\right]$ and

$$
\frac{G\left(R_{1}, 1\right)}{G(1,1)}<c<1
$$

In analogy with Section 2 we set

$$
K_{p}=\left\{u \in H_{r}^{1}\left(B_{1}\right):\left(\left|B_{1}\right|^{-1} \int_{B_{1}}|u|^{p+1}\right)^{\frac{1}{p+1}}=1 \text { and }|u| \leq c \text { in } B_{R_{1}}\right\},
$$

and we define correspondingly $J_{p}$ and $J_{\infty}$. Thanks to Lemma [5.1, it is possible to proceed as in Sections 3 and 4 with minor changes. This provides the existence of a nonconstant solution of the Neumann problem for $p$ sufficiently large.

Proof of Theorem 1.2. It is sufficient to show that $\bar{r}=1$ is a local minimum point of $F(r)$, for every choice of $V(|x|) \geq 0, V \not \equiv 0$. To this aim we compute the derivatice of $F$ as follows:

$$
F^{\prime}(r)=-\left|\partial B_{1}\right| \cdot \frac{\xi^{\prime}(r) \zeta(r)+\xi(r) \zeta^{\prime}(r)}{(\xi(r) \zeta(r))^{2}}=-\left|\partial B_{1}\right| \cdot \frac{\xi^{\prime}(r) \zeta(r) \cdot 1+\xi(r) \zeta^{\prime}(r) \cdot 1}{(\xi(r) \zeta(r))^{2}}
$$

Using (6.2) we have that $1=r^{n-1} \xi^{\prime}(r) \zeta(r)-r^{n-1} \xi(r) \zeta^{\prime}(r)$, which, substituted in the previous equality, gives

$$
F^{\prime}(r)=r^{n-1}\left|\partial B_{1}\right| \cdot \frac{\left(\xi(r) \zeta^{\prime}(r)\right)^{2}-\left(\xi^{\prime}(r) \zeta(r)\right)^{2}}{(\xi(r) \zeta(r))^{2}} .
$$


Now, the boundary condition gives $\zeta^{\prime}(1)=0$, whereas 6.2 at $r=1$ implies $\xi^{\prime}(1) \zeta(1) \neq 0$. Therefore

$$
\lim _{r \rightarrow 1^{-}} F^{\prime}(r)=-\left|\partial B_{1}\right| \cdot \frac{\left(\xi^{\prime}(1) \zeta(1)\right)^{2}}{(\xi(1) \zeta(1))^{2}}<0,
$$

which concludes the proof.

\section{Appendix}

We collect some properties of the Green function $G(r, s)$.

Lemma 6.1. Let $V(|x|) \geq 0, V \not \equiv 0$ be a smooth radial function in $B_{1}$. There exist linearly independent solutions $\xi, \zeta \in C^{2}((0,1])$ of the equation

$$
-u^{\prime \prime}(r)-\frac{n-1}{r} u^{\prime}(r)+V(r) u(r)=0, \quad u>0,
$$

satisfying $\xi^{\prime}(0)=\zeta^{\prime}(1)=0$ and enjoying the additional property

$$
\xi^{\prime}(r) \zeta(r)-\xi(r) \zeta^{\prime}(r) \equiv \frac{1}{r^{n-1}}, \quad r \in(0,1] .
$$

The same result holds in the case of Dirichlet boundary conditions at $r=1$, that is, $\xi^{\prime}(0)=\zeta(1)=0$.

Proof. In case of Dirichlet boundary conditions the result is proved by Catrina in 8, Appendix. Let us adapt the proof to the case of Neumann boundary conditions. Let $s=r^{2-n}$ and $u(r)=\tilde{u}\left(r^{2-n}\right), V(r)=\tilde{V}\left(r^{2-n}\right)$. Then (6.1) transforms into

$$
-\tilde{u}^{\prime \prime}(s)+\frac{s^{\frac{2 n-2}{2-n}}}{(n-2)^{2}} \tilde{V}(s) \tilde{u}(s)=0, \quad s \in[1, \infty) .
$$

Catrina provides, via an approximation method, a positive function $\varphi(s)$ which satisfies

$$
\varphi(s)=1+\int_{s}^{\infty}\left(1-\frac{s}{t}\right) \frac{\tilde{V}(t)}{(n-2)^{2}} \varphi(t) t^{\frac{n}{2-n}} d t
$$

and hence solves (6.3). The function $\xi(r)=\varphi\left(r^{2-n}\right) /(n-2)$ solves (6.1), and, moreover, as shown in [8], $\lim _{r \rightarrow 0^{+}} \xi^{\prime}(r)=0$. Next we set

$$
\psi(s)=\varphi(s)\left\{-\frac{1}{\varphi(1) \varphi^{\prime}(1)}+\int_{1}^{s} \frac{1}{\varphi^{2}(t)} d t\right\},
$$

which is well defined since

$$
\varphi^{\prime}(1)=\varphi(1)-1=\int_{1}^{\infty}\left(1-\frac{1}{t}\right) \frac{\tilde{V}(t)}{(n-2)^{2}} \varphi(t) t^{\frac{n}{2-n}} d t>0,
$$

by the assumption $V \geq 0, V \not \equiv 0$. A direct calculation shows that

$$
\psi^{\prime}(1)=0 \quad \text { and } \quad \varphi(s) \psi^{\prime}(s)-\varphi^{\prime}(s) \psi(s) \equiv 1, \quad s \in[1, \infty) .
$$

Hence the pair $\zeta(r)=\psi\left(r^{2-n}\right), \xi(r)$ satisfies (6.2), and the lemma is proved.

A straightforward consequence of this result is the following factorization of the Green function. 
Lemma 6.2. In the assumptions of the previous lemma it holds that

$$
G(r, s)= \begin{cases}s^{n-1} \xi(r) \zeta(s) & \text { for } r \leq s \\ s^{n-1} \xi(s) \zeta(r) & \text { for } r>s\end{cases}
$$

according to the choice of the boundary conditions.

Lemma 6.3. Let $\bar{r}$ be a local minimum point of the function $F(r)$. Then $\bar{r}$ is a strict local minimum point.

Proof. The previous lemma gives $F(r)=\left|\partial B_{1}\right| /[\xi(r) \zeta(r)]$, which is constant in an interval $(a, b)$ if and only if

$$
\xi(r)=\frac{C}{\zeta(r)} \quad \text { in }(a, b)
$$

for some positive constant $C$ (recall that $\xi$ and $\zeta$ are strictly positive). However, this cannot happen because of (6.2).

\section{ACKNOWLEDGEMENTS}

The authors wish to thank Susanna Terracini for the fruitful discussion. Work was partially supported by MIUR, Project Metodi Variazionali ed Equazioni Differenziali Non Lineari.

\section{REFERENCES}

[1] Adimurthi and G. Mancini, The Neumann problem for elliptic equations with critical nonlinearity, Nonlinear analysis, Sc. Norm. Super. di Pisa (1991), 9-25. MR1205370 (94d:35043)

[2] Adimurthi, F. Pacella and S.L. Yadava, Interaction between the geometry of the boundary and positive solutions of a semilinear Neumann problem with critical nonlinearity, J. Funct. Anal. 113 (1993), 318-350. MR1218099(94e:35030)

[3] Adimurthi and S.L. Yadava, Critical Sobolev exponent problem in $\mathbb{R}^{n}(n \geq 4)$ with Neumann boundary condition, Proc. Indian Acad. Sci. Math. Sci. 100 (1990), 275-284. MR.1081711 (91k:35013)

[4] Adimurthi and S. L. Yadava, Existence and nonexistence of positive radial solutions of Neumann problems with critical Sobolev exponents. Arch. Rational Mech. Anal. 115(3) (1991), 275-296. MR1106295(92e:35069)

[5] Adimurthi and S. L. Yadava, On a conjecture of Lin-Ni for a semilinear Neumann problem. Trans. Amer. Math. Soc. 336(2) (1993), 631-637. MR.1156299(93f:35073)

[6] A. Bahri and J. M. Coron, On a nonlinear elliptic equation involving the critical Sobolev exponent: The effect of the topology of the domain, Comm. Pure Appl. Math. 41 (1988), 253-294. MR929280 (89c:35053)

[7] H. Brezis and L. Nirenberg, Positive solutions of nonlinear elliptic equations involving critical Sobolev exponents, Comm. Pure Appl. Math. 36 (1983), 437-477. MR709644 (84h:35059)

[8] F. Catrina, A note on a result of M. Grossi, Proc. Amer. Math. Soc. 137, 11 (2009), 37173724. MR2529879 (2010i:35122)

[9] M. Comte and M. C. Knaap, Existence of solutions of elliptic equations involving critical Sobolev exponents with Neumann boundary condition in general domains. Differential Integral Equations 4(6) (1991), 1133-1146. MR1133748 (92j:35063)

[10] M. del Pino and J. Wei, Supercritical elliptic problems in domains with small holes, Ann. Inst. H. Poincaré Anal. Non Linéaire 24, 24 (2007), 507-520. MR2334989 (2008j:35077)

[11] B. Gidas, W. M. Ni and L. Nirenberg, Symmetry of positive solutions of nonlinear elliptic equations in $R^{n}$. Mathematical analysis and applications, Part A, Adv. in Math. Suppl. Stud., 7a, Academic Press, New York-London, 1981, 369-402. MR634248 (84a:35083)

[12] D. Gilbarg and N. Trudinger, Elliptic Partial Differential Equations of Second Order. Second edition. Springer-Verlag, Berlin, 1983. MR737190(86c:35035)

[13] M. Grossi, Asymptotic behaviour of the Kazdan-Warner solution in the annulus, J. Differential Equations 223, 1 (2006), 96-111. MR2210140 (2006m:35122) 
[14] M. Grossi, Radial solutions for the Brezis-Nirenberg problem involving large nonlinearities, Jour. Funct. Anal. 254 (2008), 2995-3036. MR2418617 (2009c:35126)

[15] R. Johnson, X. B. Panand Y. Yi, Positive solutions of super-critical elliptic equations and asymptotics, Comm. Partial Differential Equations 18 (1993), 977-1019. MR1218526 (94g:35083)

[16] Chang Shou Lin and Wei-Ming Ni, On the diffusion coefficient of a semilinear Neumann problem. In Calculus of variations and partial differential equations (Trento, 1986), volume 1340 of Lecture Notes in Math., pages 160-174. Springer, Berlin, 1988. MR974610 (90d:35101)

[17] F. Merle and L. A. Peletier, Positive solutions of elliptic equations involving supercritical growth, Proc. Roy. Soc. Edinburgh Sect. A 118 (1991), 49-62. MR1113842 (92g:35074)

[18] F. Merle, L. A. Peletier and J. Serrin, A bifurcation problem at a singular limit, Indiana Univ. Math. J. 43 (1994), 585-609. MR.1291530 (95j:35024)

[19] W. M. Ni, On the positive radial solutions of some semilinear elliptic equations on $R^{n}$, Appl. Math. Optim. 9 (1983), 373-380. MR694593 (84e:35050)

[20] W. M. Ni, X. B. Pan and I. Takagi, Singular behavior of least-energy solutions of a semilinear Neumann problem involving critical Sobolev exponents, Duke Math. J. 67 (1992), 1-20. MR 1174600 (93j:35081)

[21] D. Passaseo, Existence and multiplicity of positive solutions for elliptic equations with supercritical nonlinearity in contractible domains, Rend. Accad. Naz. Sci. XL Mem. Mat. 16 (1992), 77-98. MR 1205746 (94b:35024)

[22] D. Passaseo, Nonexistence results for elliptic problems with supercritical nonlinearity in nontrivial domains, J. Funct. Anal. 114 (1993), 97-105. MR:1220984(94m:35118)

Dipartimento di Matematica, Università di Roma "La Sapienza", P. le A. Moro 2, 00185 Roma, ITALY

E-mail address: grossi@mat.uniroma1.it

Dipartimento di Matematica e Applicazioni, Università degli Studi di Milano-Bicocca, Via Bicocca Degli Arcimboldi 8 - 20126 Milano, Italy

E-mail address: benedettanoris@gmail.com 